\title{
Optical and magnetic properties of $\mathrm{CuO}$ nanowires grown by thermal oxidation
}

\author{
M. Vila, C. Díaz- Guerra ${ }^{\text {a) }}$ and J. Piqueras \\ Departamento de Física de Materiales, Facultad de Físicas, Universidad Complutense de Madrid, \\ Ciudad Universitaria s/n, E-28040 Madrid, Spain. \\ a) e-mail: cdiazgue@fis.ucm.es
}

\begin{abstract}
$\mathrm{CuO}$ nanostructures with different morphologies, such as single-crystal nanowires, nanoribbons and nanorods, have been grown by thermal oxidation of copper in the (380-900) ${ }^{\circ} \mathrm{C}$ temperature range. Cathodoluminescence spectra of the nanostructures show a band peaked at $1.31 \mathrm{eV}$ which is associated to near band gap transitions of $\mathrm{CuO}$. Two additional bands centred at about $1.23 \mathrm{eV}$ and $1.11 \mathrm{eV}$, suggested to be due to defects, are observed for nanostructures grown at high temperatures. The magnetic behaviour of nanowires with lengths in the range of several microns and diameters of (50-120) $\mathrm{nm}$ has been investigated. Hysteresis loops of the nanowires show ferromagnetic behaviour from $5 \mathrm{~K}$ to room temperature.
\end{abstract}

P.A.C.S.: 61.46.Km, 75.75.-c, 78.60.Hk, 78.67.-n 


\section{Introduction}

Nanowires and other elongated nanostructures are a subject of wide and increasing interest due to the variety of their potential applications in nanotechnology. In particular, intensive research efforts have been devoted to the synthesis and investigation of the physical and chemical properties of semiconducting oxides such as $\mathrm{ZnO}, \mathrm{SnO}_{2}, \mathrm{TiO}_{2}, \mathrm{In}_{2} \mathrm{O}_{3}$ or, to a lesser extent, $\mathrm{CuO}$. Cupric oxide $(\mathrm{CuO})$ is a semiconductor often referred as a narrow gap material with band gap of $1.2 \mathrm{eV}$ e.g. [1,2] and monoclinic structure. Larger band gaps between $1.6 \mathrm{eV}$ and about $2.1 \mathrm{eV}$ have also been reported [3-5]. This oxide has useful catalytic properties, e.g ref. 6 , and potential applications in lithium-ion batteries, gas sensing or field emission devices [7-9] and was also investigated because of its relationship with cuprate high- $\mathrm{T}_{\mathrm{c}}$ superconductors. $\mathrm{CuO}$ nanowires have been synthesized by thermal decomposition of a precursor [10], chemical routes [11] or electrospinning [12]. In addition, thermal oxidation of copper has been reported by several groups to be an efficient and simple nanowire growth method [13-17]. In this case, nanowires of different dimensions as well as larger structures are obtained as a function of the temperature and time of the oxidizing treatment. Electrical conductivity and field emission of nanowires and nanorods obtained by thermal oxidation of copper have been previously studied $[17,18]$ but other physical properties, precisely optical and magnetic properties, have been much less investigated. Optical behaviour of $\mathrm{CuO}$ nanowires has been mainly assessed by absorption techniques, while luminescence data are scarce and refer to nanowires obtained by other methods [4]. Bulk $\mathrm{CuO}$ is an antiferromagnetic material with Néel temperatures reported in the (213-230) $\mathrm{K}$ range. However, surface spins can dominate magnetization in nanostructures because of uncompensated exchange coupling. This may lead to ferromagnetic-like behaviour at low temperatures, as reported in hydrothermally synthesized $\mathrm{CuO}$ nanorods [19] or in $\mathrm{CuO}$ elongated nanoparticles [20]. In this work, $\mathrm{CuO}$ nanowires have been grown by thermal 
oxidation of compacted copper powder at different temperatures. The morphology and structure of the nanowires have been studied by X-ray diffraction (XRD), scanning electron microscopy (SEM) and high-resolution transmission electron microscopy (HRTEM), while their chemical composition was assessed by energy dispersive X-ray microanalysis (EDX). Luminescence was investigated by cathodoluminescence (CL) in the SEM and magnetic measurements were performed with a superconducting quantum interference device (SQUID).

\section{Experimental method}

$\mathrm{Cu}$ powder of $99.9 \%$ purity (Sigma-Aldrich) was used as starting material. The powder was compacted to form disks of about $2 \mathrm{~mm}$ thickness and $7 \mathrm{~mm}$ diameter. The samples were then annealed at $380{ }^{\circ} \mathrm{C}, 500{ }^{\circ} \mathrm{C}, 600^{\circ} \mathrm{C}, 700{ }^{\circ} \mathrm{C}$ or $900{ }^{\circ} \mathrm{C}$ under air flow. Oxidation of copper takes place during the thermal treatments, leading to the growth of copper oxide nanostructures on the disk surface. As will be explained below, treatments at $380{ }^{\circ} \mathrm{C}$ yield a high density of $\mathrm{CuO}$ nanowires. In order to study the influence of the annealing time on the dimensions of the obtained nanowires, the $380{ }^{\circ} \mathrm{C}$ treatments were performed between 3 and 48 hours. The duration of the treatments at higher temperatures $\left(500-900{ }^{\circ} \mathrm{C}\right)$ was 6 hours. The structure of the wires was first investigated by XRD and grazing incidence XRD with a Philips X'Pert PRO diffractometer using $\mathrm{CuK \alpha}$ radiation. The morphology of the nanostructures was investigated by SEM with a Leica 440 or an FEI Inspect S scanning electron microscope. SEM-CL measurements were carried out at $90 \mathrm{~K}$ with an electron beam energy of $20 \mathrm{keV}$. The emission was monitored in the visible range (4.0-1.55 eV) by using a Hamamatsu R428P photon counting device and in the near infrared (IR) range (1.55-0.75 eV) with a Hamamatsu R5509-43 photomultiplier. A Bruker AXS Quantax spectrometer was used for EDX measurements. HRTEM investigations were carried out with a field emission JEOL $3000 \mathrm{~F}$ microscope operating at $300 \mathrm{kV}$. Two methods were used in order to collect the $\mathrm{CuO}$ 
nanowires from the treated disks for HRTEM and magnetic measurements as well. The first one was to scratch very gently the surface of the disks with a sharp, non-conductive, plastic tweezer. The second one was to sonicate the disk in butanol, collect the suspension, and wait until the liquid was evaporated. For HRTEM investigations, drops of the solution containing the nanostructures were deposited onto carbon coated grids. TEM images and electron diffraction measurements of the products obtained following both methods showed $\mathrm{CuO}$ nanowires only. For magnetic measurements, 10 to $15 \mathrm{mg}$ of nanowires detached from the pellets were introduced in a sample capsule of the SQUID avoiding any contact with metallic tools. Hysteresis loops at $5 \mathrm{~K}$ and $300 \mathrm{~K}$ were recorded up to applied field values of $\pm 50 \mathrm{kOe}$.

\section{Results and discussion}

SEM images of the $\mathrm{Cu}$ disks before thermal treatments show an almost featureless appearance, as shown in supporting information (see figure S1 available at stacks.iop.org/ ...). After the $380{ }^{\circ} \mathrm{C}$ annealing treatments, the surface of the disks appears uniformly covered by nanowires whose density and length increase with the treatment time. Many of the wires can be described as nanoneedles with a pointed tip. Figures 1(a) and 1(b) show the nanowire distribution in samples annealed at $380{ }^{\circ} \mathrm{C}$ for 3 and 14 hours respectively. In the latter case, the highest aspect ratio of the nanowires is obtained, with diameters in the range 50-120 nm and lengths between 3 and $10 \mu \mathrm{m}$. The density of nanowires, defined as the number of wires per unit area, was estimated from high-magnification SEM micrographs. For samples treated for $3 \mathrm{~h}$ the nanowires density is $\sim 10^{8} \mathrm{~cm}^{-2}$, while it increases nearly one order of magnitude for samples treated during $48 \mathrm{~h}$. The morphology and density of the nanowires grown using the above-mentioned conditions suggests the potential use of these nanostructures as field emitters. In samples treated at higher temperatures, the nanostructures are wider and most of them have rod or ribbon shapes rather than the needle-like shape of the wires grown at 380 
${ }^{\circ} \mathrm{C}$. This is shown in the image of the structures grown after the $500{ }^{\circ} \mathrm{C}$ annealing for 6 hours [Figure 1(c)]. The morphology of the nanostructures grown in the $700-900{ }^{0} \mathrm{C}$ temperature range is also different. Groups of nanocolumns with polygonal sections and rods with diameters of about $300-500 \mathrm{~nm}$ and up to $12 \mu \mathrm{m}$ long appear grouped on the surface of the disks [figure 1(d)], although their density is lower than that of the nanowires grown at lower temperatures.

XRD patterns of the samples treated at $380{ }^{\circ} \mathrm{C}$ show peaks corresponding to $\mathrm{Cu}, \mathrm{Cu}_{2} \mathrm{O}$ and $\mathrm{CuO}$. The peaks of $\mathrm{Cu}$ have a much lower relative intensity in the grazing incidence (GIXRD) patterns, indicating that they are related to the non-oxidised, inner part of the disk. Figure 2(a) shows the GIXRD pattern of the sample shown in figure 1(b). In the XRD pattern of the samples prepared at $600{ }^{\circ} \mathrm{C}$, or at higher temperatures, only $\mathrm{Cu}_{2} \mathrm{O}$ and $\mathrm{CuO}$ peaks are observed, with dominant $\mathrm{CuO}$ peaks in grazing incidence diffractograms [figure 2(b)]. XRD measurements were also carried out in nanowires separated from the treated disks, as shown in figure 2(c). All the diffraction maxima observed in the corresponding patterns were indexed to the $\mathrm{CuO}$ monoclinic structure (JCPDS card 048-1548). As stated in the introduction, several authors have reported the growth of $\mathrm{CuO}$ nanowires by thermal oxidation of copper. The mechanism of thermal growth of nanowires in air involves the initial formation of $\mathrm{CuO}_{2}$ which acts as precursor of $\mathrm{CuO}$. The XRD observations can be then explained by the formation of $\mathrm{CuO}$ nanowires from an intermediate $\mathrm{Cu}_{2} \mathrm{O}$ layer. The stress at the $\mathrm{CuO}-\mathrm{Cu}_{2} \mathrm{O}$ interface together with diffusion processes are thought to be responsible for the growth of the $\mathrm{CuO}$ nanowires [14]. At moderate temperatures the $\mathrm{Cu}$ peaks from the substrate appear along with the peaks of the oxides while treatments at higher temperatures lead to a thicker oxide layer so that the patterns consist only of $\mathrm{CuO}$ and $\mathrm{Cu}_{2} \mathrm{O}$ peaks. The previously reported $\mathrm{CuO}$ nanowires grown by oxidation have similar morphology and phase purity to that reported here. In addition, the presence of a low density of wires after oxidation at temperatures of $600{ }^{\circ} \mathrm{C}$ and higher has been previously observed [14, 15, 21]. However, 
nanostructures such as the nanorods and nanocolumns shown in figure 1(d) have not been previously obtained by oxidation methods. In ref. 13, the wires were found by TEM to be bicrystalline, while other authors reported the single crystalline nature of their nanowires. TEM study of the nanowires grown at $380{ }^{\circ} \mathrm{C}$ and the rod-like structures grown at higher temperatures, confirm that they are single crystalline and have the $\mathrm{CuO}$ monoclinic structure. Figure 3(a) shows a low magnification image of a $70 \mathrm{~nm}$ wide nanowire grown at $380{ }^{\circ} \mathrm{C}$ for 14 hours and the corresponding selected area diffraction pattern (SAED) measured in the center of the sample. The SAED patterns did not change as the electron beam was moved along the nanostructures. Figure 3(b) shows a high-resolution micrograph of the same nanowire. The interlayer distances measured in the image, 0.275 and $0.250 \mathrm{~nm}$, are respectively consistent with those of the (110) and (002) planes in the $\mathrm{CuO}$ monoclinic structure. Extended defects such as twins were not found in HRTEM observations of our nanowires.

The distribution of the two oxide phases detected by XRD has been studied by crosssectional observation in SEM. Figure 4(a) shows the nanowires and two layers, one formed by small grains in contact with the nanowires, and another one formed by larger elongated grains. The corresponding EDX oxygen map, [figure 4(b)] shows a similar intensity in the nanowires and in the thin layer, while the layer with elongated grains shows a reduced $\mathrm{O}$ signal. Furthermore, EDX spectra recorded in different points of the cross-section reveal a decrease of the $\mathrm{O} / \mathrm{Cu}$ signal ratio for spectra measured at increasing depths, which indicates that the relative content of $\mathrm{Cu}$ increases when moving from the nanowires towards the thick layer with bigger grains (see supporting information, figure S2, available at stacks.iop.org/...). This agrees with the mechanism of $\mathrm{Cu}$ oxidation in air with a first step of $\mathrm{Cu}_{2} \mathrm{O}$ formation, which would correspond to the layer with large grains and lower oxygen content, followed by the formation of $\mathrm{CuO}$, which correspond to the thin layer with small grains and the nanowires, as also supported by our XRD measurements. Such structure, with layers of the two oxides, 
has been previously reported [17,21] and a mechanism of nanowire growth via grain boundary diffusion of $\mathrm{Cu}$ ions through the $\mathrm{Cu}_{2} \mathrm{O}$ layer was proposed [17].

$\mathrm{CuO}$ band gap, and in general its optical properties, have been sometimes studied by optical absorption with a dispersion of results as the reported values of $1.85 \mathrm{eV}$ in thin films [3], $2.03 \mathrm{eV}$ [5] and $1.2 \mathrm{eV}$ [2] in nanowires, or $2.43 \mathrm{eV}$ in nanoparticles [22]. Luminescence techniques, which are important tools to investigate electronic transitions in semiconductors including band edge or near band edge transitions - have been seldom used in the case of $\mathrm{CuO}$. Lin et al. [4] estimated from photoluminescence measurements a band gap of $1.67 \mathrm{eV}$ in nanofibers. The lack of luminescence data for $\mathrm{CuO}$ is probably due to the low emission efficiency of this material. For this reason, CL in SEM, which involves a high excitation by the electron beam, appears suitable to investigate electronic recombination in $\mathrm{CuO}$. Figure 5(a) shows the CL spectrum of several samples treated at $380{ }^{\circ} \mathrm{C}$ for 30 hours. As described above, the upper layer of this sample consists of $\mathrm{CuO}$ grains and is uniformly covered by a high density of $\mathrm{CuO}$ nanowires. A single $\mathrm{CL}$ band peaked at about $1.31 \mathrm{eV}$ is observed in samples treated at this temperature for different times, as well as in CL spectra of samples grown at $500{ }^{\circ} \mathrm{C}$. However, the emission widens towards the low energy side in samples grown at $700{ }^{\circ} \mathrm{C}$ or higher temperatures. A representative example of the asymmetrical CL spectral distribution measured in these samples is shown in figure 5(b). The spectrum corresponds to a pellet treated at $900{ }^{\circ} \mathrm{C}$ for 6 hours, and appears peaked at $1.31 \mathrm{eV}$. Gaussian deconvolution reveals that actually three CL bands, respectively centred at $1.33 \mathrm{eV}, 1.23 \mathrm{eV}$ and $1.11 \mathrm{eV}$, contribute to the observed emission. In addition, it should be mentioned that CL in the visible spectral range was not detected in the present work. $\mathrm{Cu}_{2} \mathrm{O}$ shows intense luminescence - peaked near $2.16 \mathrm{eV}$ at $77 \mathrm{~K}$ - related to excitonic transitions [23], which strongly suggests that the $\mathrm{Cu}_{2} \mathrm{O}$ layer beneath the $\mathrm{CuO}$ layer and the nanowires do not contribute to the observed CL emission. In order to verify this assumption, we recorded CL spectra from nanowires separated from the treated disks. The spectral distribution of the 
emission was found to be very similar to that observed in the treated pellets covered by nanowires, although with a lower signal to noise ratio (see supporting information, figure $\mathrm{S} 3$, available at stacks.iop.org/...). We suggest that the $1.33 \mathrm{eV}$ band, whose energy is close to the band gap value reported in several absorption measurements, corresponds to near band edge transitions of $\mathrm{CuO}$. The bands peaked at lower energies would correspond to defect levels. Previous spectrally resolved photocurrent measurements [24] actually revealed the existence of recombination centres lying $1.17 \mathrm{eV}$ below the conduction band edge in $\mathrm{CuO}$ samples with a band gap of $1.35 \mathrm{eV}$. Nevertheless, little information is available on the $\mathrm{CuO}$ defect structure [25]. $\mathrm{CuO}$ is intrinsically a p-type semiconductor due to the existence of $\mathrm{Cu}$ vacancies. The formation of this point defect is favoured by growth at low temperatures, while sintering at $700-900{ }^{\circ} \mathrm{C}$ has been reported [26] to decrease nonstoichiometry. However, our CL bands centred at 1.23 and $1.11 \mathrm{eV}$ were only observed in samples treated at temperatures higher than $500{ }^{\circ} \mathrm{C}$. Furthermore, recent theoretical calculations indicate that although $\mathrm{Cu}$ vacancies are the most stable defects in $\mathrm{CuO}$, they do not introduce new states within the band gap $[27,28]$. Hence, $\mathrm{Cu}$ vacancies might not be involved in the mentioned emissions. On the contrary, calculations reveal that such states can be introduced by oxygen vacancies and antisite defects [28,29]. The formation energy of $\mathrm{O}_{\mathrm{Cu}}$ antisite defects of acceptor nature is actually only slightly higher than the formation energy of $\mathrm{Cu}$ vacancies [27]. In any case, it is currently recognized [25] that a systematic study of the stoichiometry and defect structure of $\mathrm{CuO}$, particularly in samples grown in a wide range of well-defined equilibrium conditions, is still necessary.

Samples grown at $380^{\circ} \mathrm{C}$, whose surface was completely covered by nanowires, were used for magnetic measurements. The nanowires were detached from the pellet surface, and several pellets were needed to obtain enough nanowires to perform the measurements. In $\mathrm{CuO}$, a transition from a paramagnetic to an incommensurate antiferromagnetic (AFM) state takes place near $\mathrm{T}_{\mathrm{N}}=230 \mathrm{~K}$, followed by a first-order transition to a commensurate AFM state 
near $213 \mathrm{~K}$ [30]. However, surface spins in nanostructures can lead to a net magnetic moment because of lower coordination and uncompensated exchange couplings. This effect has been observed in nanorods and nanoneedles synthesized by hydrothermal methods [31,32] and in nanoparticles prepared by chemical routes $[20,33]$. The size and shape of the nanostructures is an important parameter related to the appearance of ferromagnetic behaviour. In the case of nanoparticles, weak ferromagnetism in $\mathrm{CuO}$ was reported for sizes below $10 \mathrm{~nm}[33,34]$ while it was observed in nanorods of $40 \mathrm{~nm}$ in diameter [32], which was explained by a higher specific area. In this work, we report on the magnetic behaviour of our nanowires obtained by thermal oxidation. The hysteresis loops of the nanowires at $5 \mathrm{~K}$ and $300 \mathrm{~K}$ (figure 6) show ferromagnetic behaviour with coercive fields of 340 Oe and 60 Oe respectively. It is remarkable that the nanowires have larger sizes, $50-120 \mathrm{~nm}$ in diameter and $3-10 \mu \mathrm{m}$ in length, than the above-mentioned $\mathrm{CuO}$ nanostructures with ferromagnetic behaviour. This shows, as suggested in [32], that a large aspect ratio influences the appearance of weak ferromagnetism, which is observed in the relatively long nanowires of this work. In fact, our $\mathrm{CuO}$ nanowires possess larger coercive fields at room temperature $(60 \mathrm{Oe})$ than $\mathrm{CuO}$ nanoparticles investigated in previous works (10 Oe) [33], but smaller ones than those measured by Xiao et al. [32] in $\mathrm{CuO}$ nanorods grown by hydrothermal methods (175 Oe). These differences have been attributed [32] to shape anisotropy, which enhances coercivity. While spherical nanoparticles do not have any net shape anisotropy, shape anisotropy increases with the axial ratio of the rod (modelled as a prolate spheroid).

Due to the uncompensated surface spins, a ferromagnetic-antiferromagnetic interface is probably formed and the surface spins influence the order of the core spins via exchange coupling. Because of the large exchange interaction between $\mathrm{Cu}^{2+}$ ions [35], the short-range ferromagnetic ordering between uncompensated surface spins and the short-range ordering of the spins in the core of the nanowires extends up to $300 \mathrm{~K}$. 


\section{Conclusions}

Single crystalline $\mathrm{CuO}$ nanowires, ribbon-shaped nanostructures as well as nanorods with different morphologies have been grown by thermal oxidation of copper powder in the $380-900{ }^{\circ} \mathrm{C}$ temperature range. $\mathrm{CL}$ spectra of the nanostructures show a band peaked at 1.31 $\mathrm{eV}$, which is associated to band gap or near band gap transitions of $\mathrm{CuO}$. In the case of structures grown at high temperatures, the emission band is broader with components at 1.23 $\mathrm{eV}$ and $1.11 \mathrm{eV}$ suggested to be due to defects. Magnetic behaviour of nanowires with lengths in the range of several microns and 50-120 $\mathrm{nm}$ in diameters has been studied. Hysteresis loops of the nanowires show a ferromagnetic behaviour at low and room temperatures, with coercive fields of $340 \mathrm{Oe}$ and $60 \mathrm{Oe}$ at $5 \mathrm{~K}$ and $300 \mathrm{~K}$ respectively.

\section{Acknowledgements}

This work was supported by MEC through projects MAT2006-01259 and MAT2009-07882. 


\section{References}

[1] Rakshani A E 1986 Solid State Electron. 297

[2] Hansen B J, Lu G and Chen J, 2008 Journal of Nanomaterials 2008 Article ID 830474

[3] Santra K, Sarkar C K, Mukherjee M K and Gosh B 1992 Thin Solid Films 213226

[4] Lin H H, Wang C Y, Shih H C, Chen J M and Hsieh C T 2004 J. Appl. Phys. 955889

[5] Kaur M, Muthe K P, Despande S K, Choudhury S, Singh J B, Verma N, Gupta S K and Yakhmi J V 2006 J. Crystal Growth 289670

[6] Reitz J B and Solomon E I 1998 J. Am. Chem. Soc. 12011467

[7] Hsieh C T, Chen J M, Lin H H and Shih H C 2003 Appl. Phys. Lett. 833383

[8] Poizot P, Laruelle S, Grugeon S, Dupont L and Tarascon J M 2000 Nature 407496

[9] Wang C, Fu X Q, Xue X Y, Wang Y G and Wang T H 2007 Nanotechnology 18145506

[10] Xu C K, Liu Y K, Xu G D and Wang G H 2002 Mater. Res. Bull. 372365

[11] Wang W, Liu Z, Liu Y, Xu C, Zheng C and Wang G 2003 Appl. Phys. A 76417

[12] Wu H, Lin D D and Pan W 2006 Appl. Phys. Lett. 89133125

[13] Jiang X, Herricks T, Xia Y 2002 Nano Lett. 21333

[14] Kumar A, Srivastava A K, Tiwari P and Nandedkar R V 2004 J. Phys.: Condens. Matter 168531

[15] Xu C H, Woo C H and Shi S Q 2004 Chem. Phys. Lett. 39962

[16] Zhang K, Rossi C, Tenailleau C, Alphonse P and Chane-Ching J 2007 Nanotechnology 18275607

[17] Gonçalves A M B, Campos L C, Ferlauto A S and Lacerda R G 2009 J. Appl. Phys. 106 034303

[18] Zhu Y W, Yu T, Cheong F C, Xu X J, Lim C T, Tan V B C, Thong J T L and Sow C H 2005 Nanotechnology 1688

[19] Xiao H M, Zhu L P, Liu X M and Fu S Y 2007 Solid State Commun. 141431 
[20] Narsinga Rao G, Yao Y D and Chen J W 2005 IEEE Trans. Mag. 413409

[21] Huang L S, Yang S G, Li T, Gu B X, Du Y W, Lu Y N and Shi S Z 2004 J. Cryst.

Growth 260130

[22] Wang H, Xu J Z, Zhu J J and Chen H Y 2002 J. Crystal Growth 24488

[23] Ito T and Masumi T 1997 J. Phys. Soc. Jpn. 662185

[24] Koffyberg F P and Benko F A 1982 J. Appl. Phys. 531173

[25] Carel C, Mouallem-Bahout M and Gaudé J 1999 Solid State Ionics 11747

[26] Jeong Y K and Choi G M 1996 J. Phys. Chem. Sol. 5781

[27] Wu Dangxin, Zhang Qiming and Tao Meng 2006 Phys. Rev. B 73235206

[28] Wu Dangxin 2005 Master Thesis, University of Texas

[29] Mäki-Jaskari M A 2006 Modelling Simul Mater. Sci. Eng. 14207

[30] Ziolo J, Borsa F, Corti M, Rigamonti A and Parmigiani F 1990 J. Appl. Phys. 675864

[31] Dar M A, Kim Y S, Kim W B, Sohn J M and Shin H S 2008 Appl. Surf. Sci. 2547477

[32] Xiao H M, Zhu L P, Liu X M and Fu S Y 2007 Solid State Commun. 141431

[33] Punnoose A, Magnone H and Seehra M S 2001 Phys. Rev. B 64174420

[34] Zheng X G, Mori T, Nishiyama K, Higemoto W and Xu C N 2004 Solid State Commun. 132493

[35] Kondo O, Ono M, Sugiura E, Sugiyama K and Date M 1988 J. Phys. Soc. Jpn. 573293 


\section{Figure Captions}

Figure 1. SEM images of $\mathrm{CuO}$ nanostructures obtained at different temperatures. Nanowires grown on samples treated at $380{ }^{\circ} \mathrm{C}$ for $3 \mathrm{~h}$ (a) and $14 \mathrm{~h} \mathrm{(b),} \mathrm{(c)} \mathrm{nanoribbons} \mathrm{obtained} \mathrm{at} 500$ ${ }^{0} \mathrm{C}$ for $6 \mathrm{~h},(\mathrm{~d})$ nanocolumns grown on samples treated at $900{ }^{0} \mathrm{C}(6 \mathrm{~h})$.

Figure 2. GIXRD patterns of a disk treated at $380{ }^{\circ} \mathrm{C}$ for $30 \mathrm{~h}$ (a) and at $600{ }^{0} \mathrm{C}$ for $6 \mathrm{~h}$ (b). In both cases, measurements were carried out in samples containing a high density of nanostructures. (c) XRD pattern of nanowires separated from the treated disks. All the diffraction maxima correspond to the $\mathrm{CuO}$ monoclinic structure.

Figure 3. (a) TEM micrograph of a single-crystal $\mathrm{CuO}$ nanowire grown at $380{ }^{\circ} \mathrm{C}$. The inset shows the corresponding SAED pattern taken along the [001] zone axis. (b) HRTEM image of the same nanowire showing the (110) and (002) interplanar distances of this material.

Figure 4. (a) Cross-section SEM image of a disk treated at $380^{\circ} \mathrm{C}$ for $14 \mathrm{~h}$. The surface is covered by a high density of nanowires stemming from a thin layer of small grains grown above a thicker layer of bigger, elongated, grains. (b) EDX oxygen map of the same region showing a reduced signal from the mentioned thick layer.

Figure 5. (a) CL spectra of samples grown at $380{ }^{\circ} \mathrm{C}(30 \mathrm{~h})$, uniformly covered by a high density of $\mathrm{CuO}$ nanowires. (b) $\mathrm{CL}$ spectrum of a sample treated at $900{ }^{\circ} \mathrm{C}(6 \mathrm{~h})$. Gaussian deconvolution shows emission bands centred at 1.33, 1.23, and $1.11 \mathrm{eV}$. Circles represent the experimental data while the upper solid line corresponds to the best-fit curve.

Figure 6. Detail of the low applied field region of hysteresis loops of $\mathrm{CuO}$ nanowires measured at $5 \mathrm{~K}$ and $300 \mathrm{~K}$. The complete cycles are shown in the corresponding insets. 


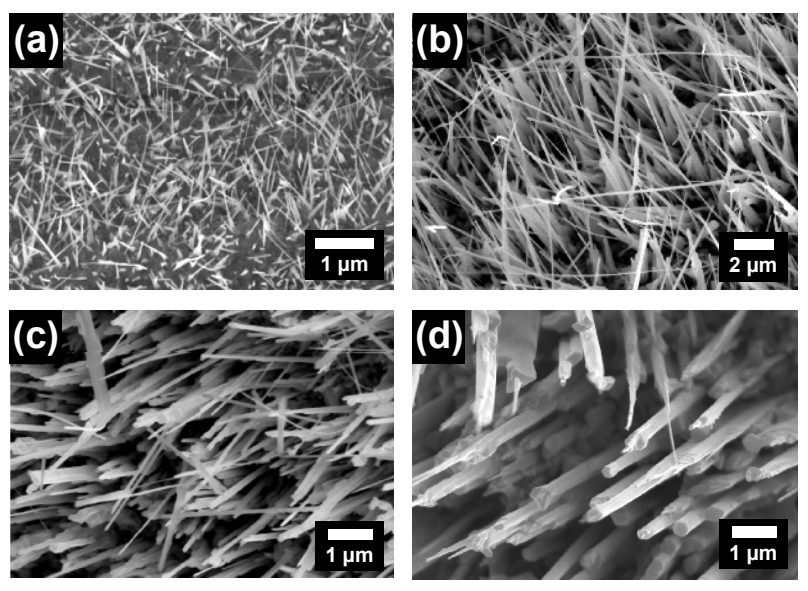

Figure 1 (Vila et al., J. Phys. D:Appl. Phys.) 

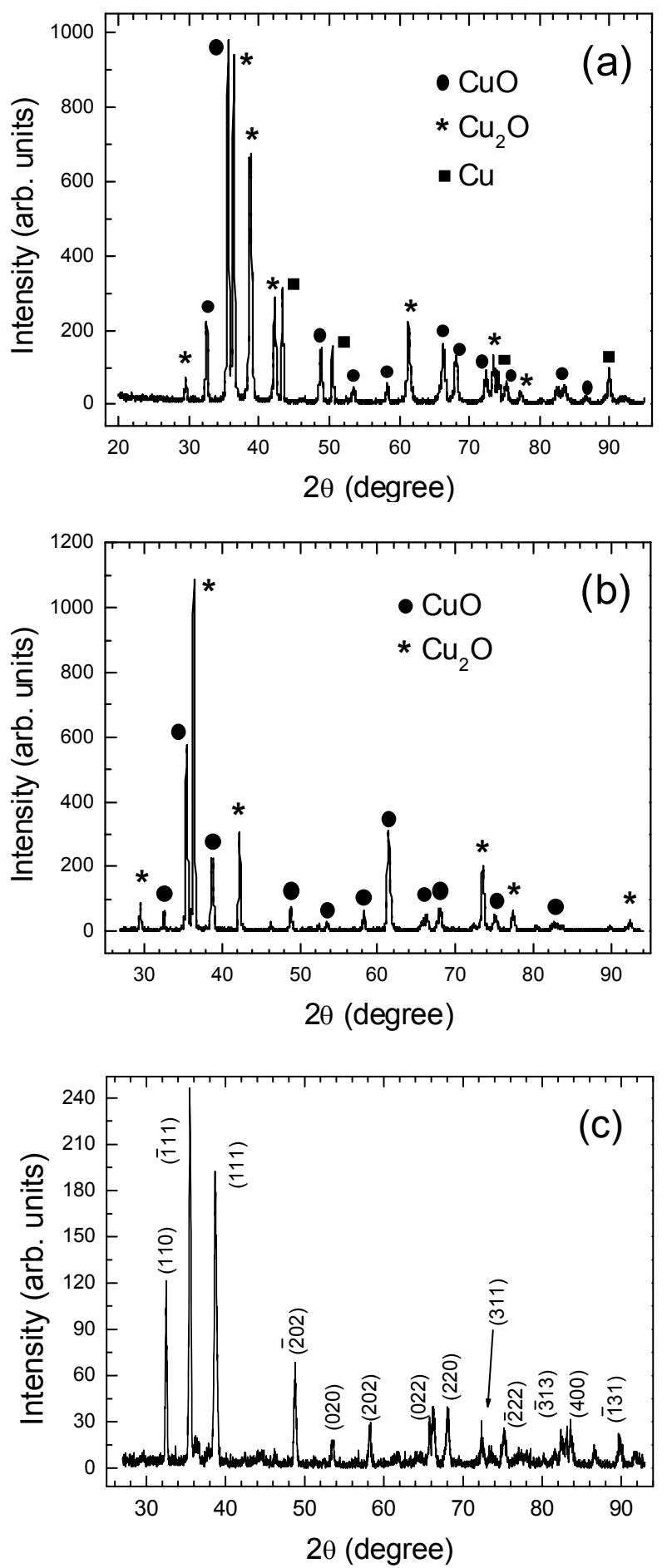

Figure 2 (Vila et al., J. Phys. D:Appl. Phys.) 


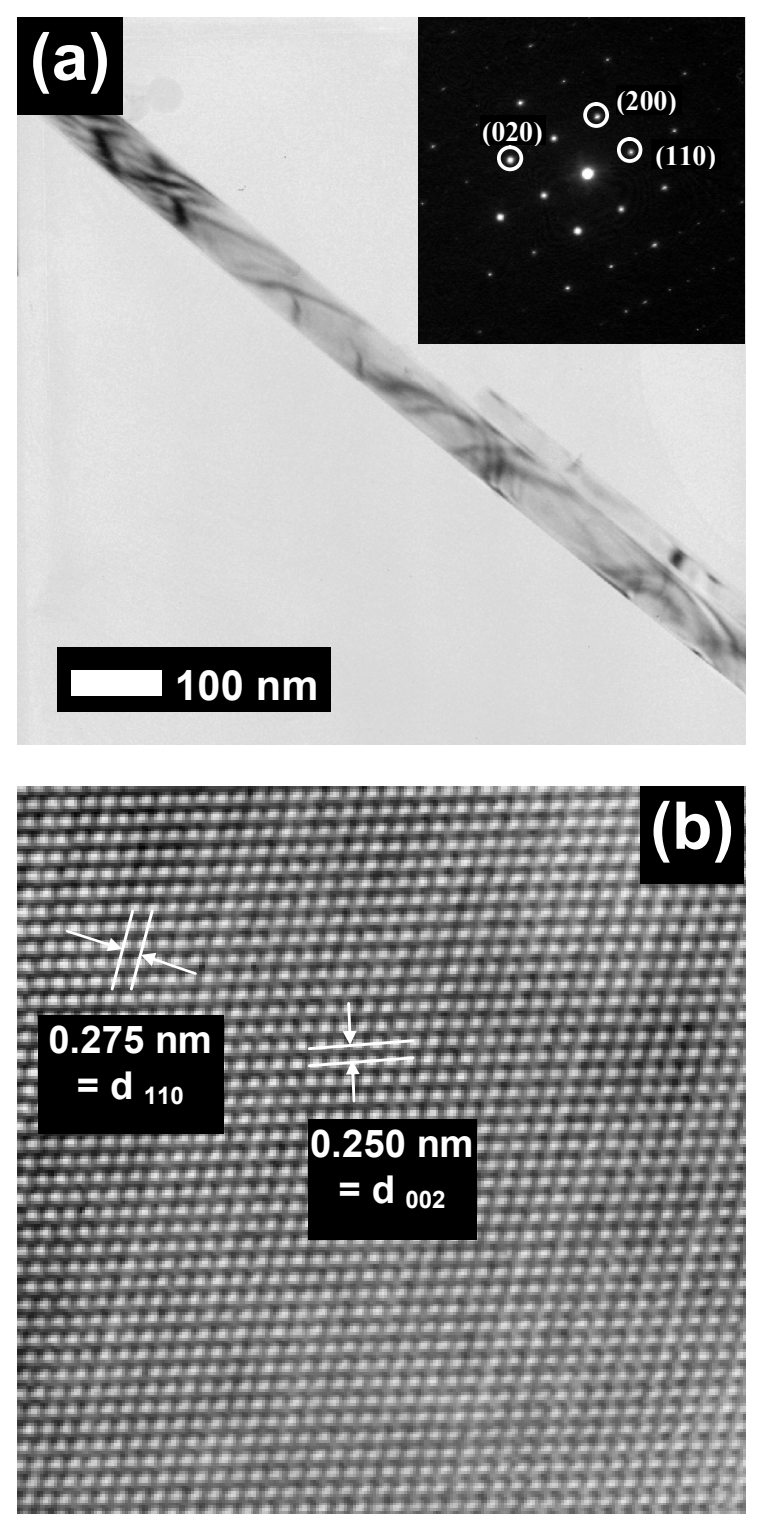

Figure 3 (Vila et al., J. Phys. D:Appl. Phys.) 

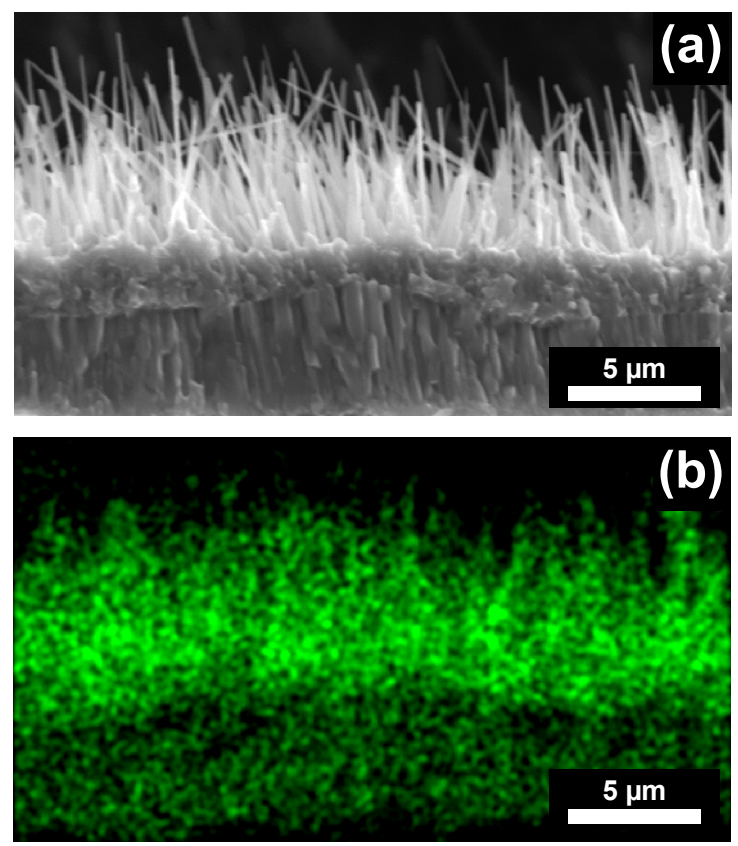

Figure 4 (Vila et al., J. Phys. D:Appl. Phys.) 

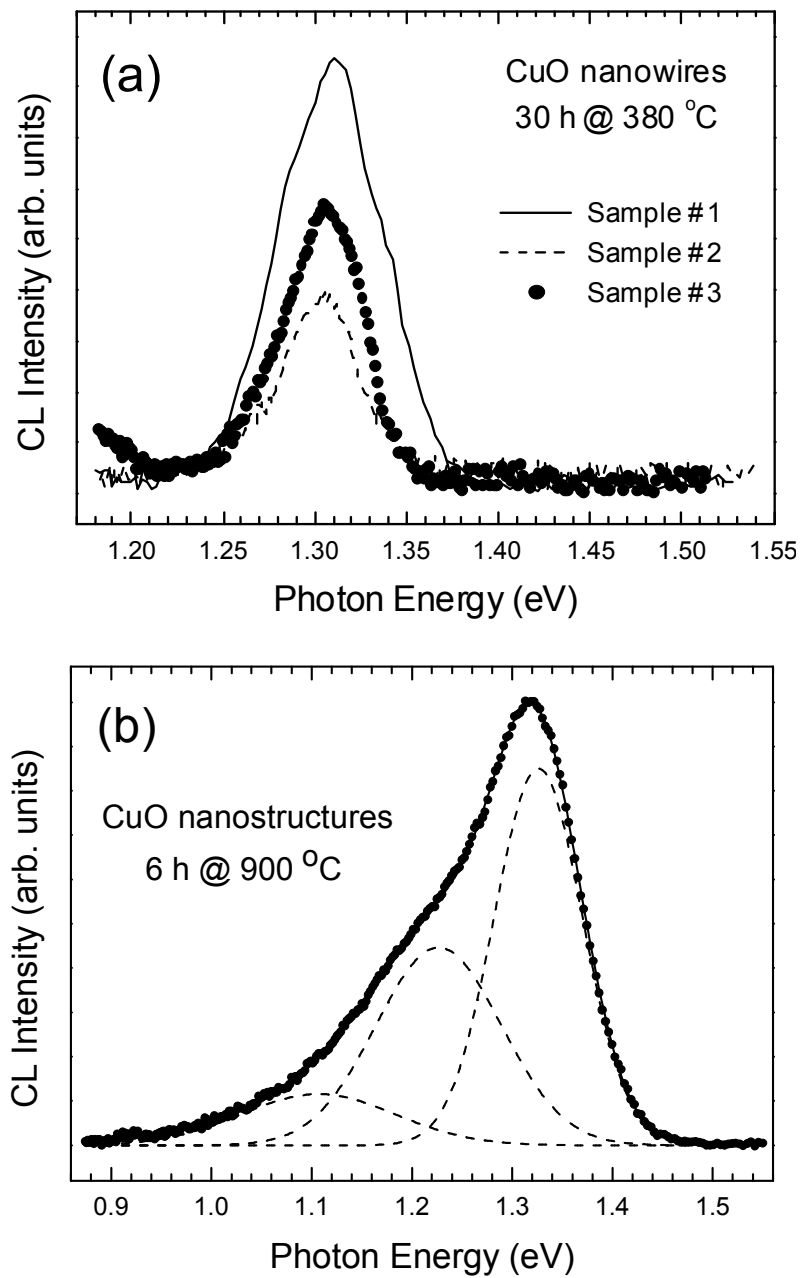

Figure 5 (Vila et al., J. Phys. D:Appl. Phys.) 

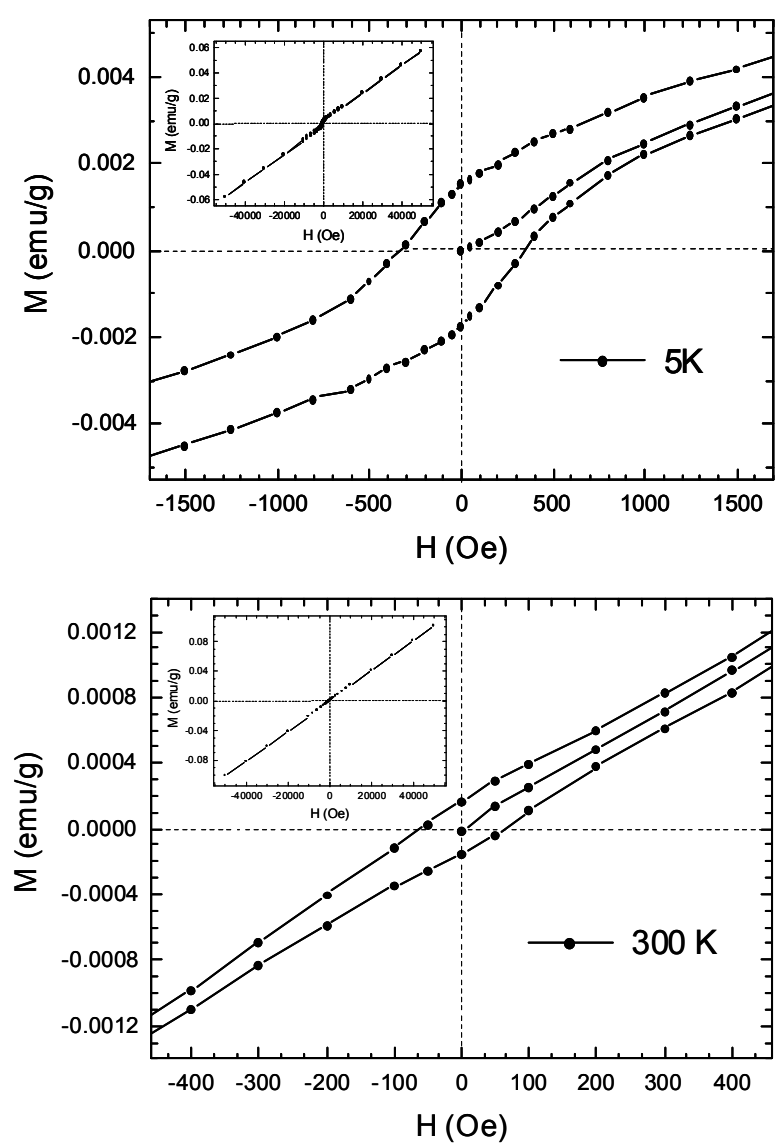

Figure 6 (Vila et al., J. Phys. D:Appl. Phys.) 